\title{
Scaling-Base Drive Function Projective Synchronization between Different Fractional-Order Chaotic Systems
}

\author{
Ping Zhou ${ }^{1,2}$ and Kun Huang ${ }^{1,2}$ \\ ${ }^{1}$ Center of System Theory and Its Applications, Chongqing University of Posts and Telecommunications, \\ Chongqing 400065, China \\ ${ }^{2}$ Key Laboratory of Industrial Internet of Things \& Networked Control, Ministry of Education, \\ Chongqing University of Posts and Telecommunications, Chongqing 400065, China
}

Correspondence should be addressed to Ping Zhou; zhouping@cqupt.edu.cn

Received 14 September 2013; Accepted 11 November 2013

Academic Editor: Jianquan Lu

Copyright (C) 2013 P. Zhou and K. Huang. This is an open access article distributed under the Creative Commons Attribution License, which permits unrestricted use, distribution, and reproduction in any medium, provided the original work is properly cited.

\begin{abstract}
A new function projective synchronization scheme between different fractional-order chaotic systems, called scaling-base drive function projective synchronization (SBDFPS), is discussed. In this SBDFPS scheme, one fractional-order chaotic system is chosen as scaling drive system, one fractional-order chaotic system is chosen as base drive systems, and another fractional-order chaotic system is chosen as response system. The SBDFPS technique scheme is based on the stability theory of nonlinear fractionalorder systems, and the synchronization technique is theoretically rigorous. Numerical experiments are presented and show the effectiveness of the SBDFPS scheme.
\end{abstract}

\section{Introduction}

In the past twenty years, many synchronization schemes for chaotic systems have been presented [1-9]. However, the function projective synchronization (FPS) scheme for chaotic systems is extensively considered due to its potential applications in secure communication. Because the drive and response systems could be synchronized with a scaling function matrix in FPS, the unpredictability of the scaling function matrix in FPS scheme can enhance the security in secure communication. In FPS, only two chaotic systems (one drive system and one response system) are considered, and the function matrix comes from one drive system. Therefore, more than one drive system (two or three drive systems or four drive systems, etc.) and one response system in FPS, and the scaling function matrix coming from multidrive systems, are general case. Moreover, multidrive systems in FPS scheme can additionally enhance the security of communication; this is due to the fact that the transmitted signals can be split into several parts, and each part can be loaded in different drive systems, or the transmitted signals can be divided time into different intervals, and the signals in different intervals can be loaded in different drive systems [8].

Motivated by the previous part, we demonstrated a new function projective synchronization scheme between different fractional-order chaotic systems in this paper, which is called scaling-base drive function projective synchronization (briefly denoted by SBDFPS). In SBDFPS scheme, there are two drive systems, which are called the scaling drive system and the base drive system, respectively. The proposed SBDFPS technique is based on the stability theory of nonlinear fractional-order systems and is theoretically rigorous. The SBDFPS between two-driver chaotic systems (fractionalorder Lorenz chaotic system as scaling drive system and fractional-order Lu chaotic system as base drive system) and one response chaotic system (fractional-order Chen chaotic system) is achieved. Numerical experiments show the effectiveness of the SBDFPS scheme.

This paper is organized as follows. In Section 2, the SBDFPS scheme between different fractional-order chaotic systems is demonstrated. In Section 3, some examples are considered and show the effectiveness of the SBDFPS scheme. Finally, the conclusion ends the paper in Section 4. 


\section{The Scaling-Base Drive Function Projective Synchronization (SBDFPS) between Different Fractional-Order Chaotic Systems}

The Caputo definition of the fractional derivative is used, which is

$$
\begin{array}{r}
D^{q} f(t)=\frac{1}{\Gamma(m-q)} \int_{0}^{t} \frac{f^{(m)}(\tau)}{(t-\tau)^{q+1-m}} d \tau, \\
m-1<q<m,
\end{array}
$$

where $D^{q}$ is called the Caputo operator, $m$ is the first integer which is not less than $q$, and $f^{(m)}(t)$ is the $m$-order derivative for $f(t)$; that is, $f^{(m)}(t)=d^{m} f(t) / d t^{m}$.

Now, the SBDFPS scheme between different fractionalorder chaotic systems will be established. Consider the fractional-order scaling drive chaotic system and base drive chaotic system and one response chaotic system described by systems (2), (3), and (4), respectively as follows:

$$
\begin{gathered}
D^{q_{d 1}} x_{1}=f_{d 1}\left(x_{1}\right), \\
D^{q_{d 2}} x_{2}=f_{d 2}\left(x_{2}\right), \\
D^{q_{r}} y=f_{r}(y)+M\left(x_{1}, x_{2}, y\right),
\end{gathered}
$$

where $0<q_{d i}<1(i=1,2)$ and $0<q_{r}<1$ are fractional-order. $x_{1}=\left(x_{11}, x_{12}, \ldots, x_{1 n}\right)^{T}, x_{2}=\left(x_{21}, x_{22}, \ldots, x_{2 n}\right)^{T}$, and $y=$ $\left(y_{1}, y_{2}, \ldots y_{n}\right)^{T}$ are state vectors of fractional-order chaotic systems (2)-(4). $f_{d i}(i=1,2): R^{n} \rightarrow R^{n}$ and $f_{r}: R^{n} \rightarrow R^{n}$ are differential nonlinear functions. $M\left(x_{1}, x_{2}, y\right) \in R^{n \times 1}$ is a vector controller and will be designed.

Definition 1. Give the scaling drive system (2), the base drive systems (3), and the response system (4). It is said to scalingbase drive function projective synchronization (SBDFPS) if there exist real nonzero constant matrix $C_{i} \in R^{n \times n}(i=1,2)$ and nonzero scaling function matrix $S_{i}\left(x_{i}\right) \in R^{n \times n}(i=1,2)$ such that

$$
\lim _{t \rightarrow+\infty}\|e\|=\lim _{t \rightarrow+\infty}\left\|\left[C_{1} S_{1}\left(x_{1}\right)+C_{2} S_{2}\left(x_{2}\right)\right] x_{1}-y\right\|=0,
$$

where $\|\cdot\|$ represents the Euclidean norm.

Remark 2. If $C_{1} \neq 0, C_{2}=0$, then the SBDFPS scheme will be turned into FPS. If $C_{i}=0(i=1,2)$, then the SBDFPS scheme will be turned into a chaos control problem.

Remark 3. System (2) and systems (3) in SBDFPS scheme may be integer order systems. So, the SBDFPS between integer order chaotic system and fractional-order can be achieved.

Let the SBDFPS error between the scaling drive system (2), base drive systems (3), and response system (4) be defined as

$$
e=y-\left[C_{1} S_{1}\left(x_{1}\right)+C_{2} S_{2}\left(x_{2}\right)\right] x_{1},
$$

where $e=\left(e_{1}, e_{2}, \ldots, e_{n}\right)^{T}$.
Now, choose vector controller $M\left(x_{1}, x_{2}, y\right) \in R^{n \times 1}$ as

$$
\begin{aligned}
M & \left(x_{1}, x_{2}, y\right) \\
= & D^{q_{r}}\left\{\left[C_{1} S_{1}\left(x_{1}\right)+C_{2} S_{2}\left(x_{2}\right)\right] x_{1}\right\} \\
& \quad-f_{r}\left\{\left[C_{1} S_{1}\left(x_{1}\right)+C_{2} S_{2}\left(x_{2}\right)\right] x_{1}\right\}+M_{1}\left(x_{1}, x_{2}, y\right) e,
\end{aligned}
$$

where feedback controller $M_{1}\left(x_{1}, x_{2}, y\right) \in R^{n \times n}$ will be designed later.

By (6) and (7), system (4) can be changed as follows: $D^{q_{r}} e$

$$
=f_{r}(y)-f_{r}\left\{\left[C_{1} S_{1}\left(x_{1}\right)+C_{2} S_{2}\left(x_{2}\right)\right] x_{1}\right\}+M_{1}\left(x_{1}, x_{2}, y\right) e .
$$

In this paper, we assume that

$$
f_{r}(y)-f_{r}\left\{\left[C_{1} S_{1}\left(x_{1}\right)+C_{2} S_{2}\left(x_{2}\right)\right] x_{1}\right\}=M_{2}\left(x_{1}, x_{2}, y\right) e,
$$

where $M_{2}\left(x_{1}, x_{2}, y\right) \in R^{n \times n}$. In fact, many fractional-order chaotic (hyperchaotic) systems satisfy this assumption.

By (9), system (8) can be rewritten as

$$
D^{q_{r}} e=\left[M_{1}\left(x_{1}, x_{2}, y\right)+M_{2}\left(x_{1}, x_{2}, y\right)\right] e .
$$

By (10), the SBDFPS between the scaling drive system (2), base drive systems (3), and response system (4) is turned into the following problem: select suitable $M_{1}\left(x_{1}, x_{2}, y\right) \in R^{n \times n}$ such that the system (10) asymptotically converges to zero.

Theorem 4. Select suitable matrix $M_{1}\left(x_{1}, x_{2}, y\right) \in R^{n \times n}$ such that $M_{1}\left(x_{1}, x_{2}, y\right)+M_{2}\left(x_{1}, x_{2}, y\right)$ satisfy the following conditions:

$$
\begin{aligned}
& \text { (1) } M_{i j}=-M_{j i}(i \neq j) \\
& \text { (2) } M_{i i} \leq 0 \text { (all } M_{i i} \text { are not equal to zero), }
\end{aligned}
$$

where $M_{i j}\left(i, j=1,2, \ldots n, \forall M_{i j} \in R\right)$ are the entries of $M_{1}\left(x_{1}\right.$, $\left.x_{2}, y\right)+M_{2}\left(x_{1}, x_{2}, y\right)$. Then the SBDFPS between the scaling drive system (2), base drive systems (3), and response system (4) can be reached.

Proof. Let $\lambda$ be one of the eigenvalues of matrix $M_{1}\left(x_{1}\right.$, $\left.x_{2}, y\right)+M_{2}\left(x_{1}, x_{2}, y\right)$ and $\rho$ the corresponding nonzero eigenvector. So, we have

$$
\left[M_{1}\left(x_{1}, x_{2}, y\right)+M_{2}\left(x_{1}, x_{2}, y\right)\right] \rho=\lambda \rho .
$$

By (11), taking conjugate transpose on both sides of (11), one can obtain

$$
\overline{\left\{\left[M_{1}\left(x_{1}, x_{2}, y\right)+M_{2}\left(x_{1}, x_{2}, y\right)\right] \rho\right\}^{\mathbf{T}}}=\bar{\lambda} \rho^{\mathbf{H}},
$$

where $\mathbf{H}$ denotes conjugate transpose.

Now, (12) multiplied right by $\rho$ plus (11) multiplied left by $\rho^{\mathbf{H}}$. Thus

$$
\begin{aligned}
\rho^{\mathbf{H}}\{ & {\left[M_{1}\left(x_{1}, x_{2}, y\right)+M_{2}\left(x_{1}, x_{2}, y\right)\right] } \\
& \left.+\left[M_{1}\left(x_{1}, x_{2}, y\right)+M_{2}\left(x_{1}, x_{2}, y\right)\right]^{\mathbf{H}}\right\} \rho \\
& =\rho^{\mathbf{H}} \rho(\lambda+\bar{\lambda}) .
\end{aligned}
$$


Thus

$$
\begin{aligned}
\lambda+\bar{\lambda}=\rho^{\mathbf{H}}\{ & {\left[M_{1}\left(x_{1}, x_{2}, y\right)+M_{2}\left(x_{1}, x_{2}, y\right)\right] } \\
& \left.+\left[M_{1}\left(x_{1}, x_{2}, y\right)+M_{2}\left(x_{1}, x_{2}, y\right)\right]^{\mathbf{H}}\right\} \frac{\rho}{\rho^{\mathbf{H}} \rho} .
\end{aligned}
$$

Using $M_{i j}=-M_{j i}\left(i \neq j, \forall b_{i j} \in R\right)$, so

$$
\lambda+\bar{\lambda}=\rho^{\mathbf{H}}\left(\begin{array}{cccc}
2 M_{11} & 0 & \cdots & 0 \\
0 & 2 M_{22} & \cdots & 0 \\
0 & 0 & \cdots & 0 \\
0 & 0 & 0 & 2 M_{n n}
\end{array}\right) \frac{\rho}{\rho^{\mathbf{H}} \rho}
$$

Because $M_{i i} \leq 0$ (all $M_{i i}$ are not equal to zero), we have

$$
\lambda+\bar{\lambda} \leq 0
$$

That is,

$$
\left|\arg \lambda\left[M_{1}\left(x_{1}, x_{2}, y\right)+M_{2}\left(x_{1}, x_{2}, y\right)\right]\right| \geq 0.5 \pi
$$

Therefore,

$$
\left|\arg \lambda\left[M_{1}\left(x_{1}, x_{2}, y\right)+M_{2}\left(x_{1}, x_{2}, y\right)\right]\right| \geq q_{r} \frac{\pi}{2} .
$$

According to the stability theorem for nonlinear fractional-order systems [9-11], (18) indicates that the equilibrium point $e=(0,0, \ldots, 0)^{T}$ in system $(10)$ is asymp-totically stable; that is,

$$
\lim _{t \rightarrow+\infty}\|e\|=\lim _{t \rightarrow+\infty}\left\|\left[C_{1} S_{1}\left(x_{1}\right)+C_{2} S_{2}\left(x_{2}\right)\right] x_{1}-y\right\|=0 .
$$

Equation (19) demonstrates that the SBDFPS between the scaling drive system (2), base drive systems (3), and response system (4) can be received. The proof is completed.

\section{Illustrative Examples}

To illustrate the effectiveness of the proposed synchronization scheme, some examples are given and the numerical simulations are yielded.

First, the improved version of Adams-Bashforth-Moulton numerical algorithm [12] for fractional-order nonlinear systems is introduced. Now, consider the nonlinear fractionalorder system

$$
\begin{aligned}
& \frac{d^{q_{1}} z_{1}}{d t^{q_{1}}}=h_{1}\left(z_{1}, z_{2}\right), \\
& \frac{d^{q_{2}} z_{2}}{d t^{q_{2}}}=h_{2}\left(z_{1}, z_{2}\right),
\end{aligned}
$$

with initial condition $\left(z_{1}(0), z_{2}(0)\right)$. Let $\tau=T / N$ and $t_{n}=n \tau$ $(n=0,1,2 \ldots, N)$. Then, nonlinear fractional-order system (20) is discretized as follows:

$$
\begin{aligned}
& z_{1}(n+1) \\
& =z_{1}(0)+\frac{\tau^{q_{1}}}{\Gamma\left(q_{1}+2\right)} \\
& \times\left[h_{1}\left(z_{1}^{p}(n+1), z_{2}^{p}(n+1)\right)\right. \\
& \left.+\sum_{j=0}^{n} \alpha_{1, j, n+1} h_{1}\left(z_{1}(j), z_{2}(j)\right)\right], \\
& z_{2}(n+1) \\
& =z_{2}(0)+\frac{\tau^{q_{2}}}{\Gamma\left(q_{2}+2\right)} \\
& \times\left[h_{2}\left(z_{1}^{p}(n+1), z_{2}^{p}(n+1)\right)\right. \\
& \left.+\sum_{j=0}^{n} \alpha_{2, j, n+1} h_{2}\left(z_{1}(j), z_{2}(j)\right)\right],
\end{aligned}
$$

where

$$
\begin{gathered}
z_{1}^{p}(n+1)=z_{1}(0)+\frac{1}{\Gamma\left(q_{1}\right)} \sum_{j=0}^{n} \beta_{1, j, n+1} h_{1}\left(z_{1}(j), z_{2}(j)\right), \\
z_{2}^{p}(n+1)=z_{2}(0)+\frac{1}{\Gamma\left(q_{2}\right)} \sum_{j=0}^{n} \beta_{2, j, n+1} h_{2}\left(z_{1}(j), z_{2}(j)\right), \\
\alpha_{i, j, n+1} \\
= \begin{cases}n^{q_{i}+1}-\left(n-q_{i}\right)(n+1)^{q_{i}}, & j=0 \\
(n-j+2)^{q_{i}+1}+(n-j)^{q_{i}+1}-2(n-j+1)^{q_{i}+1}, & 1 \leq j \leq n \\
1, & j=n+1,\end{cases} \\
\beta_{i, j, n+1}=\frac{\tau^{q_{i}}}{q_{i}}\left[(n-j+1)^{q_{i}}-(n-j)^{q_{i}}\right],
\end{gathered}
$$

The error of this approximation is

$$
\begin{array}{r}
\left|z_{i}\left(t_{n}\right)-z_{i}(n)\right|=o\left(\tau^{p_{i}}\right), \quad p_{i}=\min \left(2,1+q_{i}\right) \\
(i=1,2) .
\end{array}
$$




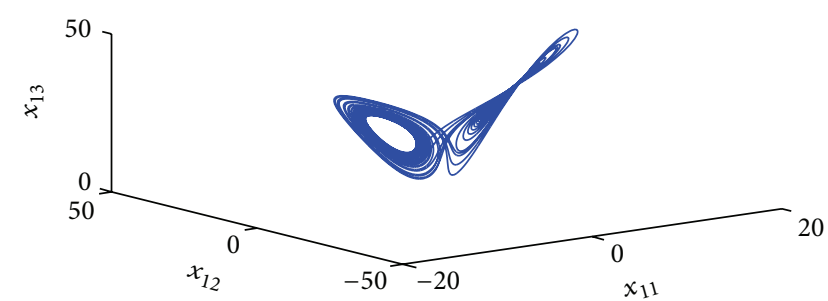

FIgURE 1: Chaotic attractors of fractional-order Lorenz system (25) for $q_{d 1}=0.994$.

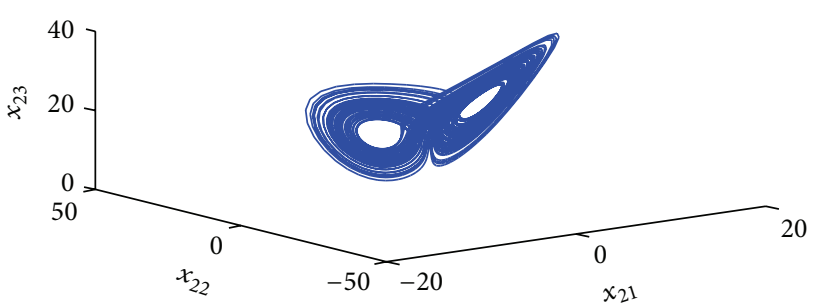

Figure 2: Chaotic attractors of fractional-order Lu system (26) for $q_{d 2}=0.95$.

The fractional-order Lorenz chaotic system [7] is depicted as

$$
\begin{gathered}
D^{q_{d 1}} x_{11}=10\left(x_{12}-x_{11}\right), \\
D^{q_{d 1}} x_{12}=28 x_{11}-x_{12}-x_{11} x_{13}, \\
D^{q_{d 1}} x_{13}=x_{11} x_{12}-\frac{8 x_{13}}{3} .
\end{gathered}
$$

The fractional-order Lorenz system (25) exhibits chaotic behavior for fractional-order $q \geq 0.993$. The chaotic attractor for $q_{d 1}=0.994$ is shown in Figure 1 . as

The fractional-order Lu chaotic system [13] is described

$$
\begin{aligned}
& D^{q_{d 2}} x_{21}=36\left(x_{22}-x_{21}\right), \\
& D^{q_{d 2}} x_{22}=20 x_{22}-x_{21} x_{23}, \\
& D^{q_{d 2}} x_{23}=x_{21} x_{22}-3 x_{23} .
\end{aligned}
$$

Its chaotic attractor for $q_{d 2}=0.95$ is illustrated in Figure 2 .

The fractional-order Chen chaotic system [7] is

$$
\begin{gathered}
D^{q_{r}} y_{1}=35\left(y_{2}-y_{1}\right), \\
D^{q_{r}} y_{2}=-7 y_{1}+28 y_{2}-y_{1} y_{3}, \\
D^{q_{r}} y_{3}=y_{1} y_{2}-3 y_{3} .
\end{gathered}
$$

The fractional-order Chen system (27) exhibits chaotic behavior for fractional-order $q_{r} \geq 0.83$. The chaotic attractor of fractional-order Chen system (27) for $q_{r}=0.85$ is displayed in Figure 3.

Now, the fractional-order Lorenz chaotic system (25) is selected as the scaling drive system, the fractional-order Lu chaotic system (26) is selected as the base drive system, and the fractional-order Chen chaotic system (27) is selected as

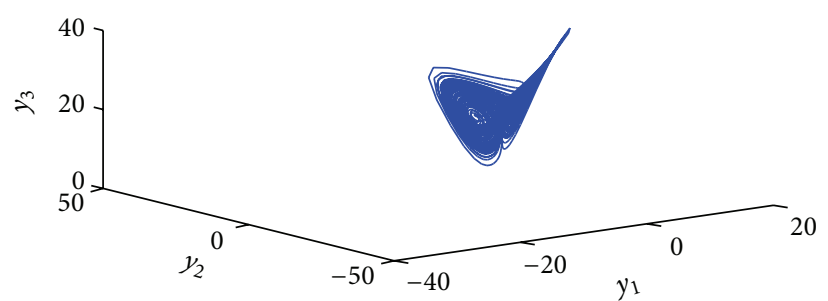

FIGURE 3: Chaotic attractors of fractional-order Chen system (27) for $q_{r}=0.85$.

response system. Our goal is to realize the SBDFPS between the scaling drive system (25), the base drive system (26), and response system (27).

According to the results in Section 2, we derive that

$$
M_{2}\left(x_{1}, x_{2}, y\right)=\left(\begin{array}{ccc}
-35 & 35 & 0 \\
-7-y_{3} & 28 & -\beta \\
y_{2} & \beta & -3
\end{array}\right) \text {, }
$$

where $\beta=x_{11}\left[\sum_{i=1}^{2} C_{i} S_{i}\left(x_{i}\right)\right]_{11}+x_{12}\left[\sum_{i=1}^{2} C_{i} S_{i}\left(x_{i}\right)\right]_{12}+$ $x_{13}\left[\sum_{i=1}^{2} C_{i} S_{i}\left(x_{i}\right)\right]_{13},\left[\sum_{i=1}^{2} C_{i} S_{i}\left(x_{i}\right)\right]_{1 j}(j=1,2)$ are the elements of matrix $\left[\sum_{i=1}^{2} C_{i} S_{i}\left(x_{i}\right)\right] . C_{i} \in R^{3 \times 3}(i=1,2)$ are real nonzero constant matrix.

Now, we select matrix $M_{1}\left(x_{1}, x_{2}, y\right)$ as follows:

$$
M_{1}\left(x_{1}, x_{2}, y\right)=\left(\begin{array}{ccc}
0 & -28+y_{3} & -y_{2} \\
0 & -30 & 0 \\
0 & 0 & 0
\end{array}\right) .
$$

Then,

$M_{1}\left(x_{1}, x_{2}, y\right)+M_{2}\left(x_{1}, x_{2}, y\right)=\left(\begin{array}{ccc}-35 & 7+y_{3} & -y_{2} \\ -7-y_{3} & -2 & -\beta \\ y_{2} & \beta & -3\end{array}\right)$.

According to Theorem 4, we can obtain the following:

$$
\left|\arg \lambda\left[M_{1}\left(x_{1}, x_{2}, y\right)+M_{2}\left(x_{1}, x_{2}, y\right)\right]\right| \geq q_{r} \frac{\pi}{2} \text {. }
$$

This result means that

$$
\lim _{t \rightarrow+\infty}\|e\|=\lim _{t \rightarrow+\infty}\left\|\left[C_{1} S_{1}\left(x_{1}\right)+C_{2} S_{2}\left(x_{2}\right)\right] x_{1}-y\right\|=0 .
$$

Equation (32) implies that the SBDFPS between the scaling drive system (25), base drive systems (26), and response system (27) can be received.

For example, let

$$
\begin{gathered}
C_{i}=\operatorname{diag}(1,1,1) \quad(i=1,2), \\
S_{1}\left(x_{1}\right)=\operatorname{diag}\left[35\left(x_{12}-x_{11}\right),-7 x_{11}-x_{11} x_{13}, x_{11} x_{12}\right], \\
S_{2}\left(x_{2}\right)=\operatorname{diag}\left[10\left(x_{22}-x_{21}\right),-x_{22}-x_{21} x_{23}, x_{21} x_{22}-x_{23}\right] .
\end{gathered}
$$



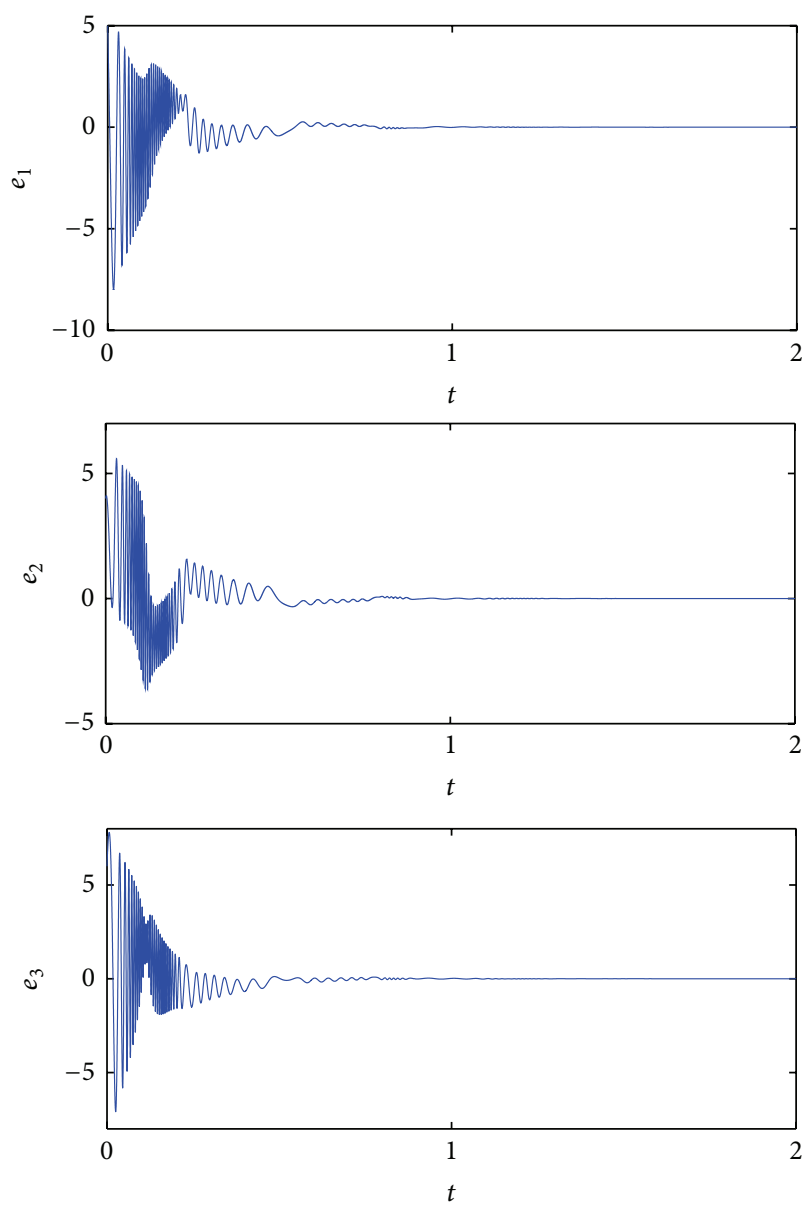

FIGURE 4: The SBDFPS errors between the scaling drive system (25), base drive systems (26), and response system (27).

The initial conditions are $\left(x_{11}, x_{12}, x_{13}\right)=(2,2,2),\left(x_{21}\right.$, $\left.x_{22}, x_{23}\right)=(1,1,1)$, and $\left(y_{1}, y_{2}, y_{3}\right)=(5,-24,8)$, respectively. The numerical experiments are illustrated in Figure 4.

\section{Conclusions}

In this paper, the scaling-base drive function projective synchronization (SBDFPS) is presented. The SBDFPS scheme is different from the FPS scheme because the scaling function matrix comes from more than one chaotic system (the scaling drive system and the base drive system). The SBDFPS between the fractional-order Lorenz chaotic system (scaling drive system), the fractional-order Lu chaotic system (base drive system), and the fractional-order Chen chaotic system (response system) is taken for example. Numerical experiments show the effectiveness of the SBDFPS scheme.

\section{Conflict of Interests}

The authors declare that there is no conflict of interests regarding the publication of this paper.

\section{References}

[1] L. M. Pecora and T. L. Carroll, "Synchronization in chaotic systems," Physical Review Letters, vol. 64, no. 8, pp. 821-824, 1990.

[2] T. L. Carroll and L. M. Pecora, "Synchronizing chaotic circuits," IEEE Transactions on Circuits and Systems I, vol. 38, pp. 453456, 1991.

[3] L. Kacarev and U. Parlitz, "Generalized synchronization, predictability, and equivalence of unidirectionally coupled dynamical systems," Physical Review Letters, vol. 76, pp. 1816-1819, 1996.

[4] L. Kacarev and U. Parlitz, "General approach for chaotic synchronization, with application to communication," Physical Review Letters, vol. 74, pp. 5028-5031, 1996.

[5] R. Mainieri and J. Rehacek, "Projective synchronization in the three-dimensional chaotic systems," Physical Review Letters, vol. 82, pp. 3042-3045, 1999.

[6] H. Y. Du, Q. S. Zeng, C. H. Wang, and M. X. Ling, "Function projective synchronization in coupled chaotic systems," Nonlinear Analysis. Real World Applications, vol. 11, no. 2, pp. 705-712, 2010.

[7] P. Zhou and W. Zhu, "Function projective synchronization for fractional-order chaotic systems," Nonlinear Analysis. Real World Applications, vol. 12, no. 2, pp. 811-816, 2011.

[8] J. W. Sun, Y. Shen, Q. Yin, and C. J. Xu, "Compound synchronization of four memristor chaotic oscillator systems and secure communication," Chaos, vol. 23, no. 1, Article ID 013140, 2013.

[9] E. Ahmed, A. M. A. El-Sayed, and H. A. A. El-Saka, "Equilibrium points, stability and numerical solutions of fractionalorder predator-prey and rabies models," Journal of Mathematical Analysis and Applications, vol. 325, no. 1, pp. 542-553, 2007.

[10] Z. Odibat, "A note on phase synchronization in coupled chaotic fractional order systems," Nonlinear Analysis. Real World Applications, vol. 13, no. 2, pp. 779-789, 2012.

[11] A. S. Hegazi, E. Ahmed, and A. E. Matouk, "On chaos control and synchronization of the commensurate fractional order Liu system," Communications in Nonlinear Science and Numerical Simulation, vol. 18, no. 5, pp. 1193-1202, 2013.

[12] C. P. Li and G. J. Peng, "Chaos in Chen's system with a fractional order," Chaos, Solitons \& Fractals, vol. 22, no. 2, pp. 443-450, 2004.

[13] W. H. Deng and C. P. Li, "Chaos synchronization of the fractional Lu system,” Physica A, vol. 353, pp. 61-72, 2005. 


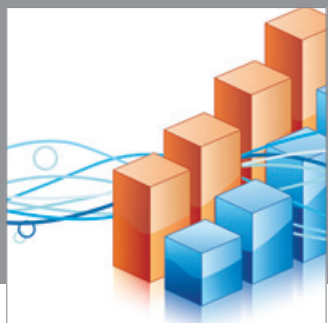

Advances in

Operations Research

mansans

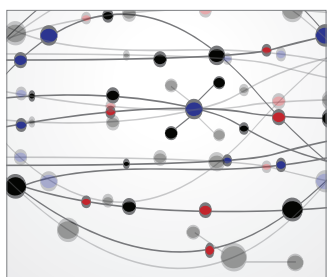

The Scientific World Journal
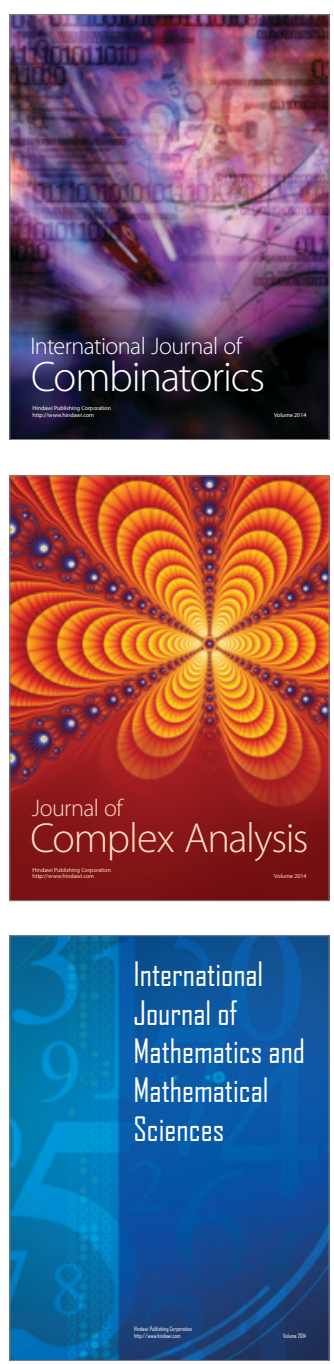
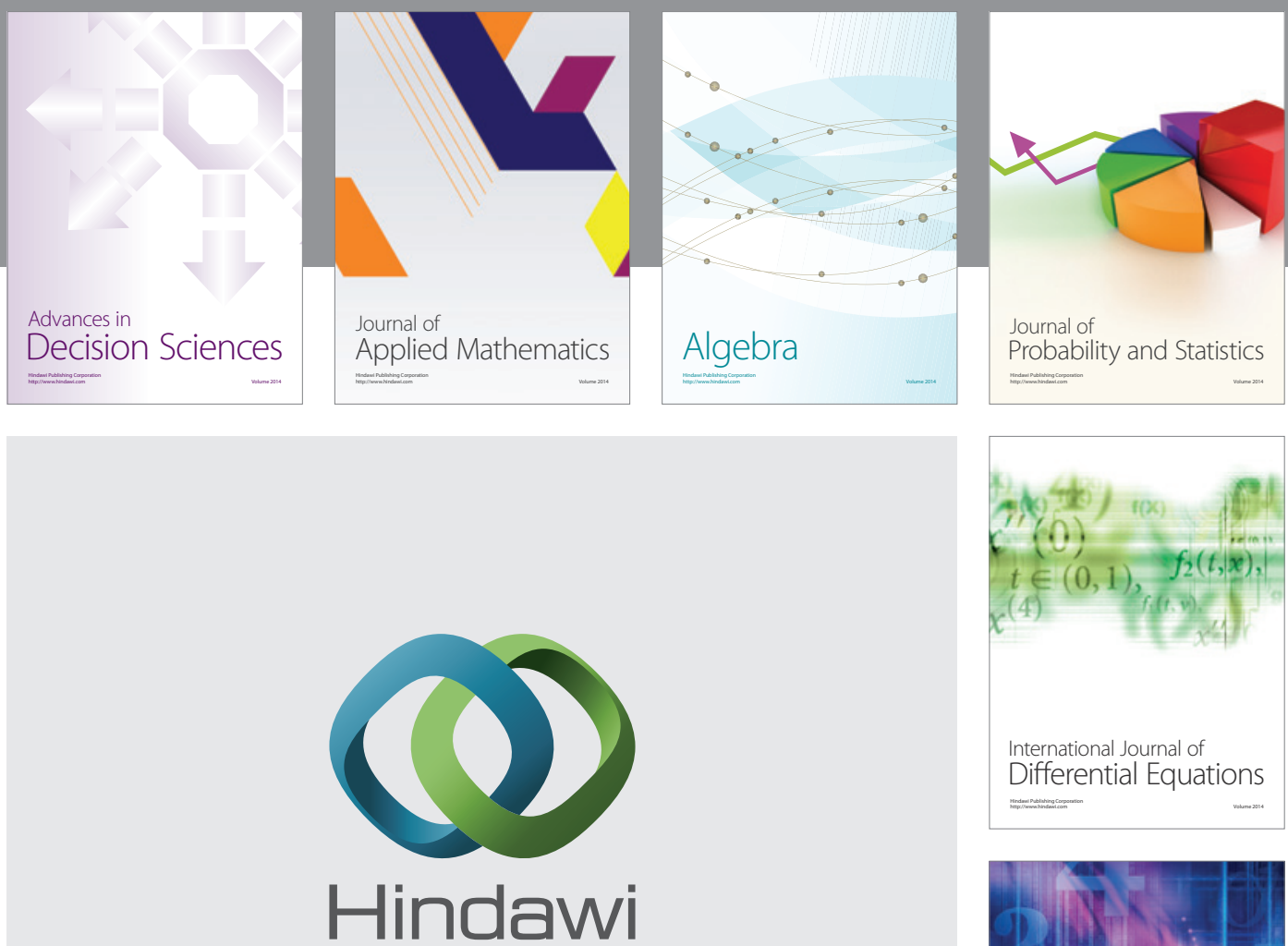

Submit your manuscripts at http://www.hindawi.com
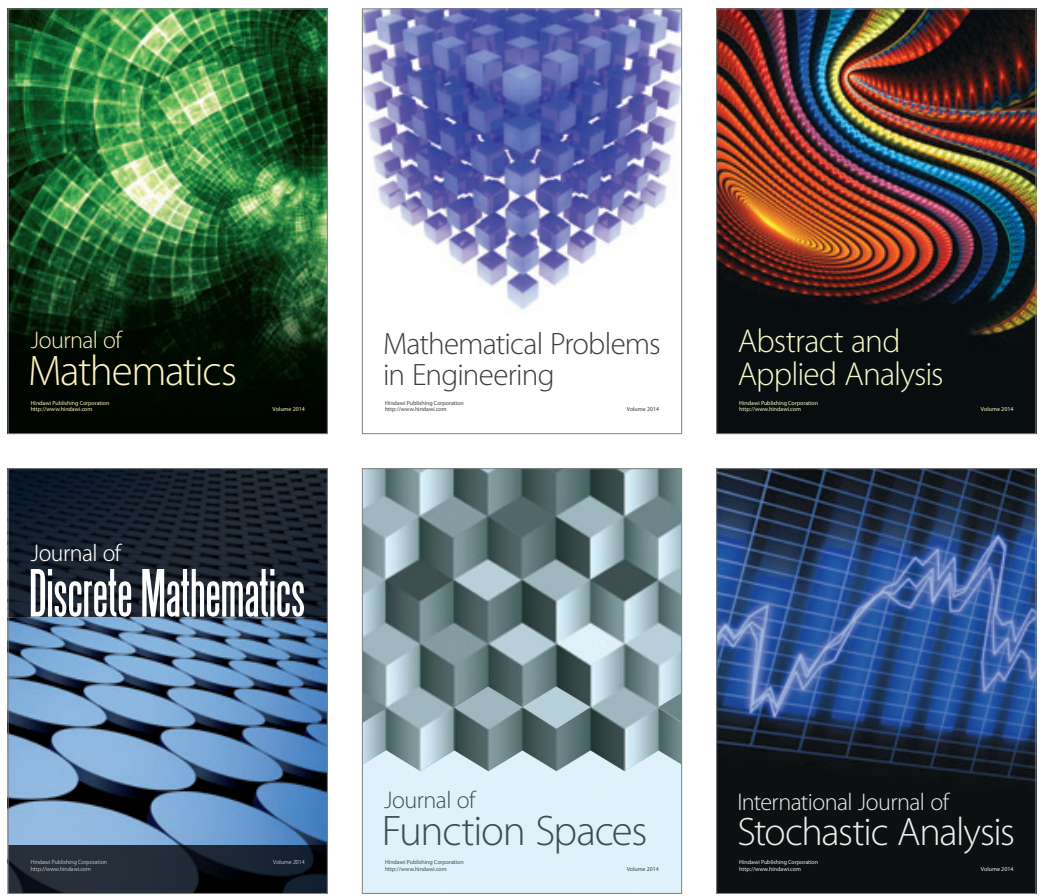

Journal of

Function Spaces

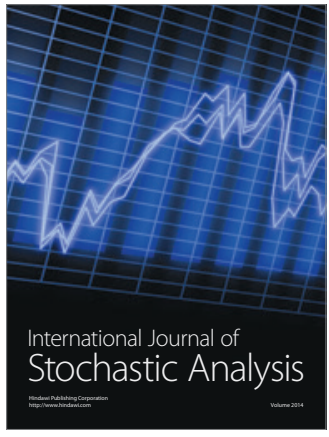

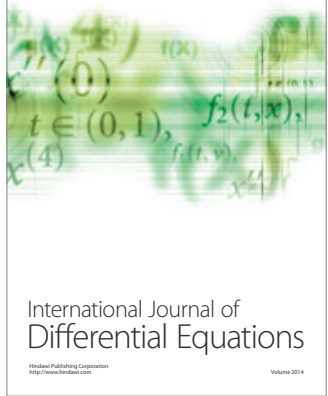
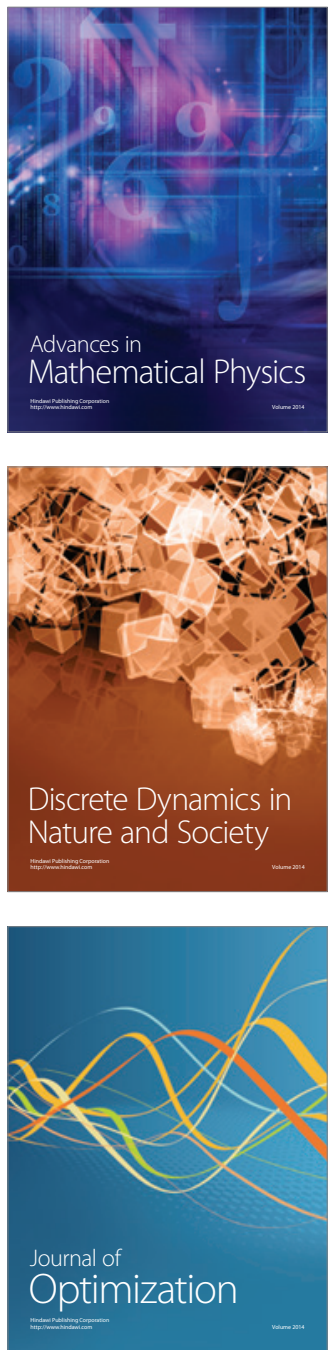